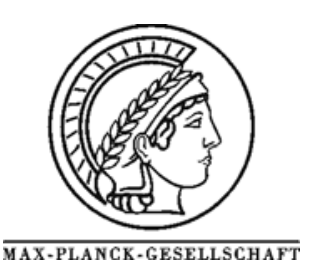

\title{
Growth behaviors of ultrathin ZnSe nanowires by Au-catalyzed molecular-beam epitaxy
}

\author{
Y. Cai ${ }^{1}$, T. L. Wong ${ }^{1}$, S. K. Chan ${ }^{1}$, I. K. Sou ${ }^{1}$, D. S. Su ${ }^{2}$, N. Wang ${ }^{1 *}$ \\ ${ }^{1}$ Department of Physics and the Institute of Nano Science and Technology, the Hong Kong University of Science \\ and Technology, Clear Water Bay, Hong Kong, China \\ ${ }^{2}$ Department of Inorganic Chemistry, Fritz-Haber Institute of the Max Planck Society, Faradayweg 4-6, \\ 14195 Berlin, Germany.
}

* Corresponding author: e-mail phwang@ust.hk,

Received 29 July 2008; accepted 23 October 2008; published 9 December 2008.

\begin{abstract}
Ultrathin ZnSe nanowires grown by Au-catalyzed molecular-beam epitaxy show an interesting growth behavior of diameter-dependence of growth rates. The smaller the nanowire diameter, the faster is its growth rate. This growth behavior is totally different from that of the nanowires with diameters greater than $60 \mathrm{~nm}$ and can not be interpreted by the classical theories of the vapor-liquid-solid mechanism. For the Au-catalyzed nanowire growth at low temperatures, we found that the surface and interface incorporation and diffusion of the source atoms at the nanowire tips controlled the growth of ultrathin ZnSe nanowires.
\end{abstract}

The unique configuration of the metal catalytic growth of nanowires (also known as the vapor-liquid-solid (VLS) growth) makes it very promising for applications in nanotechnology. The most significant work on the mechanism of the unidirectional growth of semiconductor whiskers through the VLS mechanism was published by Wagner and Ellis. ${ }^{1}$ Classically, the unidirectional growth of $\mathrm{Si}$ whiskers, for example, can be simply interpreted based on the difference of the sticking coefficients of the impinging vapor source atoms on the liquid (the catalytic droplet) and on the solid surfaces (the whisker and substrate). An ideal liquid surface captures all impinging Si source atoms, while a solid surface of Si rejects almost all source atoms if the temperature is sufficiently high. Due to the presence of metal catalysts, the geometry and atomic structure of the interface between the metal catalyst and the whisker have been found to be very critical to the whisker growth, particularly the growth velocity, growth direction or crystal orientation. In the classical VLS model, it is believed that the metal catalyst is in molten state which absorbs the source materials to form a supersaturated liquid droplet.
The precipitation of the source atoms occurs at the dropletwhisker interface, and the precipitation rate is mainly determined by the supersaturation of the droplet. Givargizov et al., ${ }^{2,3}$ determined the whisker growth rate as a function of the driving force of supersaturation $(\Delta \mu / k T)$ and first empirically described the growth rate by

$$
V=\frac{d L}{d t}=b\left(\frac{\Delta \mu_{o}}{k_{B} T}-\frac{4 \Omega \sigma}{d k_{B} T}\right)^{n}
$$

where $b$ and $n(\sim 2)$ were empirical fitting parameters. Using a two-dimensional model of island nucleation and growth $^{2,3}$, this relationship was later justified numerically and then

$$
V=V_{o} \exp \left(-\frac{\pi \Omega \eta^{2}}{3 k T h \Delta \mu}\right)
$$

where $\eta$ was the island edge energy density and $h$ the layer thickness. A typical kinetic experimental result is the growth rate dependence on the whisker diameters. The larger the whisker diameter, the faster is its growth rate. 

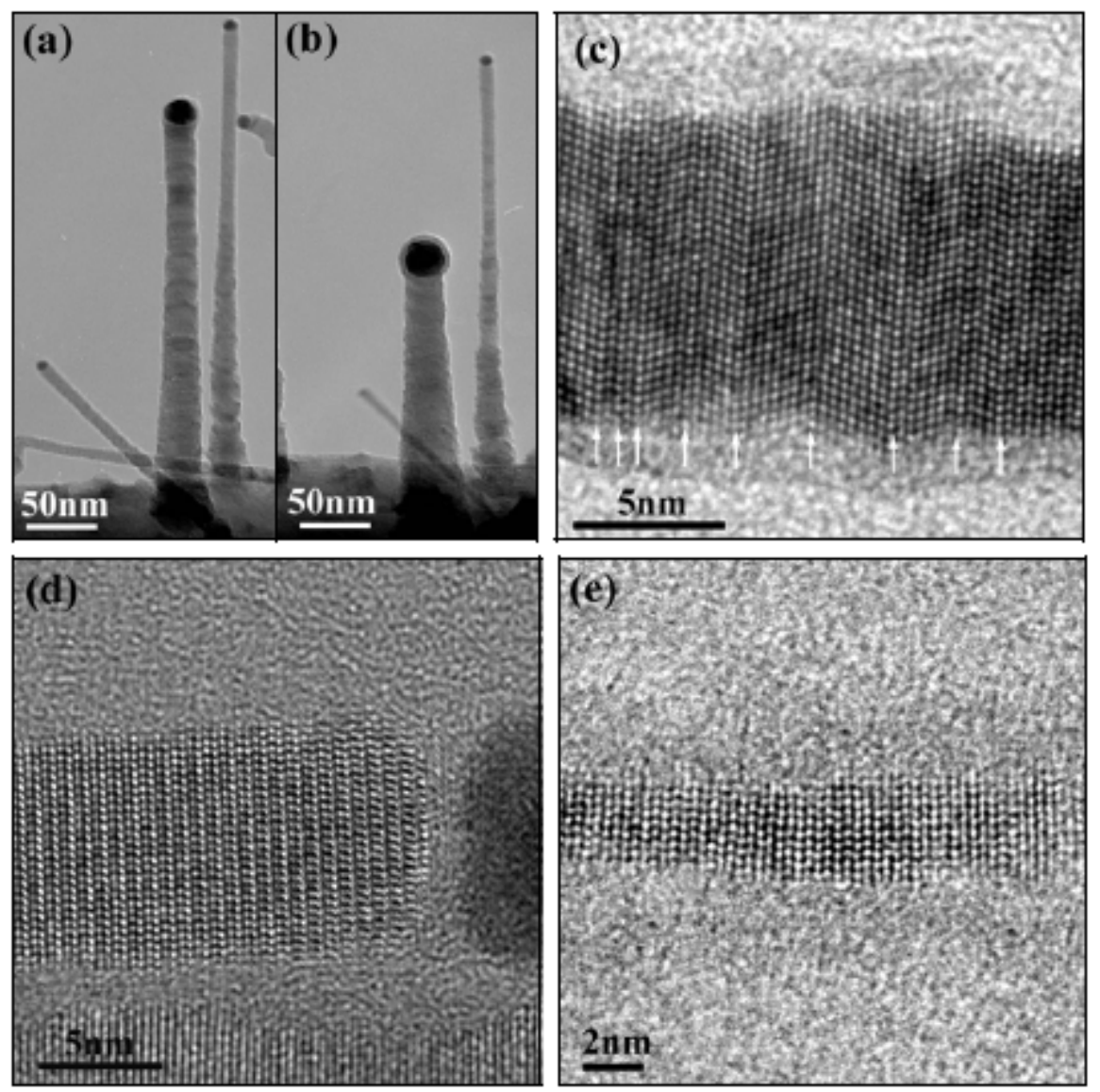

Fig. 1: (a) and (b) ZnSe nanowires with small diameters show fast growth rates. (c) High-density stacking faults and twins (marked by the arrows) frequently occur in ZnSe nanowires grown at a low temperature. (d) and (e) Ultrathin ZnSe nanowires always form HCP structure.

This growth phenomenon is attributed to the well-known Gibbs-Thomson effect, i.e., the decrease of supersaturation as a function of the whisker diameter. ${ }^{2,3}$

Due to the change of the driving force (the difference of chemical potentials), Si whiskers with small diameters $(<100 \mathrm{~nm})$ grow very slowly. Obviously, there is a critical diameter at which $\Delta \mu=0$ and the whisker growth stops completely. Those whiskers with diameters smaller than the critical diameter (about 50nm) should stop growing. This classical VLS mechanism has been extrapolated to explain the growth of most nanowires. However, in recent years, both experimentalists and theorists ${ }^{4-7}$ have demonstrated that thin semiconductor nanowires with diameter smaller than $50 \mathrm{~nm}$ can grow and show interesting growth behaviors. For example, in the growth of thin $\mathrm{Si}$ and $\mathrm{ZnSe}$ nanowires catalyzed by $\mathrm{Au}$ particles, smaller nanowires have higher growth rates compared to thicker ones, ${ }^{5-7}$ and most of ultrathin nanowires grow at relatively low temperatures. Recently, Kodambaka et al..$^{8}$ demonstrated by in-situ transmission electron microscopy (TEM) that solid catalysts led to Ge nanowire growth even at a temperature below the eutectic point. In this paper, we present the diameter-dependence of the growth rate of $\mathrm{ZnSe}$ nanowires fabricated by molecular-beam epitaxy (MBE), and the structural changes of these nanowires grown at low temperatures. We report that ultrathin nanowires display different growth behaviors compared to the classical VLS growth $^{2,3}$ or other growth models controlled by surface incorporation and diffusion mechanisms. ${ }^{9}$

ZnSe nanowires were grown by a VG V80H MBE system which was dedicated to ZnSe-based II-VI compound growth in a single chamber. A thin Au layer was deposited on a GaAs substrate at $150{ }^{\circ} \mathrm{C}$ and then annealed at $530{ }^{\circ} \mathrm{C}$ for 10 minutes in order to generate uniform $\mathrm{Au}$ nano-catalysts on the substrate surface. ZnSe nanowires were grown at different temperatures using a ZnSe compound source. Details of the experiment setup can be found in a previous paper. ${ }^{10}$ The nanowire samples were prepared by cleaving the substrates into small pieces (without any chemical pretreatment) and directly characterized by TEMs (JEOL2010F and Philips CM120).

For the VLS growth, the temperature is critical to nanowire quality and growth direction. ${ }^{11}$ As shown in Fig. 1 (a) and (b), ZnSe nanowires formed at a temperature below $390{ }^{\circ} \mathrm{C}$ have non-uniform diameters at the initial growth stage. The nanowire roots are thicker than the tips, and the surfaces near the roots are rough. This is obviously 

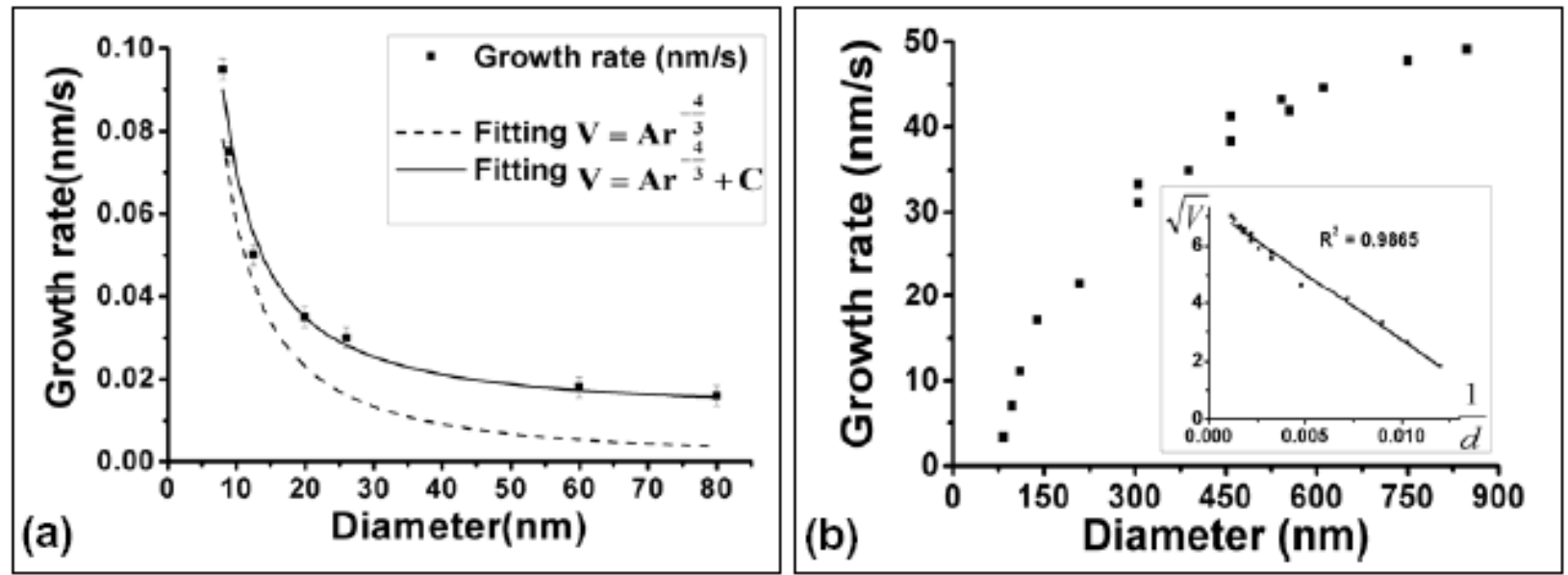

Fig. 2: (a) The solid dots indicate the growth rates measured from different diameters of ZnSe nanowires. The dashed line is the fitting curve by $d L / d t=A r^{-4 / 3}$ and the solid line by $d L / d t=A r^{-4 / 3}+C$. (b) Experimental data of the whisker growth rates reported by Givargizov (1975). ${ }^{2}$

because the deposition of $\mathrm{ZnSe}$ on the substrate surface is significant when the temperature is too low and these deposited atoms diffuse from the substrate surface to the nanowire growth fronts. ZnSe nanowires grown at a high temperature do not have this morphology. ${ }^{10}$ Once the nanowires reach a certain length, there is no obvious change in the diameters. For the tapered nanowires shown in Fig. 1 (a) and (b), the diameters may change gradually from $30 \mathrm{~nm}$ at the roots to about $5 \mathrm{~nm}$ at the tops. The quality of the thick nanowires is poor in comparison with the nanowires grown at a higher temperature $\left(>530{ }^{\circ} \mathrm{C}\right)$. A high density of stacking faults and twins always occurs in these nanowires. Fig. 1(c) illustrates the stacking faults and twining structures observed in an individual nanowire. One of the reasons for the formation of these planar defects was due to the phase transformation from the face center cubic (FCC) structure to the hexagonal close-packed (HCP) structure. The chemical composition of the source materials, e.g. the evaporation ratio of $\mathrm{Zn}$ :Se and surface energies may also result in the formation of these defects. ${ }^{12,13} \mathrm{We}$ have observed that ZnSe nanowires formed at a temperature higher than $530{ }^{\circ} \mathrm{C}$ were always a cubic structure and contained few defects. Decrease of the growth temperature could result in a high density of stacking faults, nano twins and some portion of HCP structure. As shown in Fig. 1 (d) and (e), ultrathin ZnSe nanowires with diameters of about 3-6 nm formed perfect HCP structure at $390{ }^{\circ} \mathrm{C}$.

From our measurement, the growth rate of thin ZnSe nanowires (diameters $<60 \mathrm{~nm}$ ) shows a strong diameterdependent phenomenon. Smaller nanowires have higher growth rates compared to thicker ones (see Fig. 2 (a)). This is totally different from the growth of the nanowires or whiskers with diameters greater than $100 \mathrm{~nm}$ (see Fig. 2 (b), data from Ref. 3). Figure 2 (a) illustrates the changes of growth rates versus the diameters of thin $\mathrm{ZnSe}$ nanowires. The relationship between the growth rates and the diameters can be described by $V=1 / d^{n}+C_{0}\left(C_{0}\right.$ is a con- stant and $n$ is about 1-2). Obviously, this relation does not agree with the classical VLS model, ${ }^{2,3}$ in which the metal catalysts are liquid (above the eutectic point), and the nanowire growth is determined by (i) the incorporation of the source atoms on the droplet, (ii) diffusion through the droplet and (iii) precipitation at the liquid-solid interface (See Fig. 3 (a)). From our observation, for ultrathin nanowires, however, the growth may largely deviate from these three steps and also deviate from the growth model controlled by surface incorporation and diffusion mechanisms, i.e. the source materials captured by the droplet diffuse along the droplet surface to the growth front (see Fig. 3(b). In this case, the growth rate can be described by $V=1 / r$ (see Ref. 9). This means that the growth rate is only determined by the circumference of the liquid-solid interface. Similar growth phenomena of diameter-dependence of growth rates have been observed in III-V (e.g., GaAs, $\mathrm{GaP}$, InAs and InP) nanowire growth by metal organic vapor-phase epitaxy and chemical beam epitaxy, and the growth models based on surface diffusion mechanisms

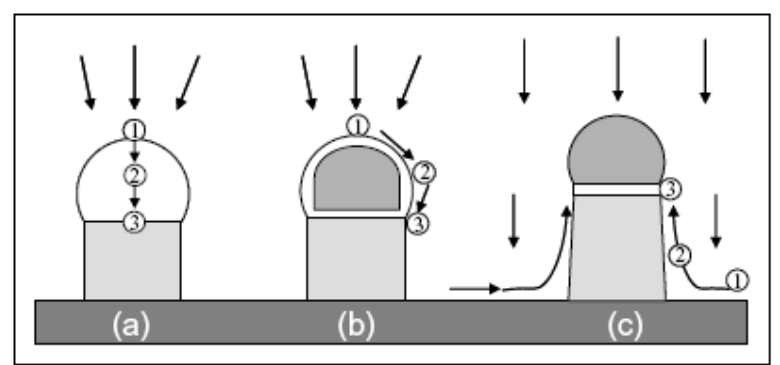

Fig. 3: Different diffusion models for the source atoms to incorporate into the growth front of the nanowire. (a) The classical VLS. (b) The metal droplet is in partially molten state. Its surface and interface are liquid, while the core of the droplet may be solid. (c) The metal catalyst is solid, but the interface is liquid. 

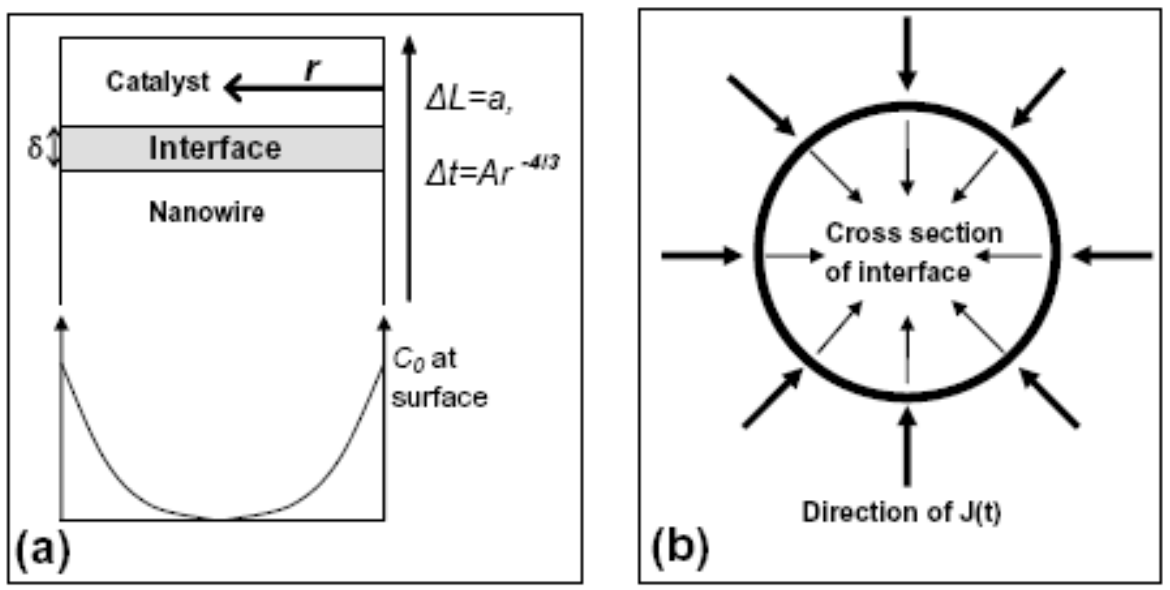

Fig. 4: (a) The schematic concentration profile of the source atoms at the catalytic interface. (b) The flux $J(t)$ of the source atoms flowing into the catalytic interface.

have been proposed. ${ }^{14,15}$ For example, to explain the diameter-dependent growth rate of InAs nanowires, Froberg, et al. ${ }^{14}$ has proposed a combined model which counts for both the Gibbs-Thomson effect and material diffusion from the substrate surface. For a nanowire with a diameter larger than $25 \mathrm{~nm}$, it is found that its growth rate is controlled by surface diffusion (the growth rate decreases with increasing the diameter). Due to the Gibbs-Thomson effect, when the diameter is smaller than $25 \mathrm{~nm}$, the growth rate decreases as the diameter shrinks. This is because the driving force for the incorporation of atoms to the catalyst is reduced with decreasing the catalyst diameter. In this case, the catalyst is considered as a droplet if the temperature is sufficiently high. For ultrathin nanowires, however, the growth temperatures are often lower than the eutectic point of the bulk material, and the catalyst may not be a droplet during growth. On the one hand, because of the nano size effect, the melting temperature of a nano-sized catalyst decreases as the size of the catalyst shrinks. But, on the other hand, due to the Gibbs-Thomson effect, the decrease of the catalyst droplet diameter lowers the solubility of the source atoms and thus shifts the melting temperature of the catalyst. For Au-semiconductor alloys (e.g., Au-Si, Au-Ge and $\mathrm{Au}-\mathrm{ZnSe}$ ), a deviation from the eutectic point generally causes the increase of the melting points. Therefore, when the growth temperature falls, the metal catalysts at the nanowire tips with relatively small diameters became solid first, while those catalysts with relatively large diameters may still remain in the liquid state. Since the growth of ultrathin nanowires can still maintain under the temperature below the eutectic point (the metal catalyst is solid) as observed by in-situ TEM, ${ }^{8}$ the real incorporation and diffusion processes of the source atoms at nanowire tips are complicated and have not been addressed so far.

We proposed a model to interpret the growth behaviors of thin nanowires by solid catalysts, and the growth is mainly controlled by surface/interface incorporation of atoms. This model is based on (i) the catalyst is solid, (ii) the source atoms deposited on the substrate surface and the nanowire surface diffuse along the nanowire side walls and preferentially flow into the catalyst interface to result in the nanowire growth, and (iii) the nanowire growth rate is mainly due to the atomic diffusion through the interface. Fig. 3 (c) shows schematically this surface/interface incorporation process. We consider the interface at the solid catalyst as a grain boundary. Since the grain boundary diffusion is slower than the surface diffusion, ${ }^{16}$ the growth of the nanowires in the present model is limited by the interface diffusion. According to the Fisher model for grain boundary diffusion ${ }^{17}$, the atom concentration at a grain boundary is described by:

$$
c=c_{0} \exp \left(-\pi^{-1 / 4} \frac{(4 D / t)^{1 / 4}}{\left(\delta D_{g b}\right)^{1 / 2}} r\right)
$$

where, $D$ is the volume diffusion coefficient, $D_{g b}$ is the grain boundary diffusion coefficient, $\delta$ is the grain boundary width $c_{0}$ is the concentration at the surface, $r$ is the radius of the nanowire (see Fig. 4 (a)). The flux of the atoms flowing into the grain boundary (Fig. 4(b)) at time $t$ is:

$$
J(t)=-D_{g b} \partial_{r} c(r, t)
$$

where the concentration is quasi-static. Assuming that $\Delta t$ is the time needed for growing $\Delta L$ (one lattice layer of $a$ ), then the total number of atoms in $\Delta L$ is:

$$
\begin{aligned}
& N=\pi r_{0}{ }^{2} a \rho= \\
& =2 \pi r_{0} \int_{0}^{\Delta t} J(t) d t=2 \pi r_{0} c_{0} \pi^{-1 / 4} \sqrt{\frac{D_{g b}}{\delta}} \int_{0}^{\Delta t} t^{-1 / 4} d t
\end{aligned}
$$

Then, 


$$
\Delta t=\left(r_{0} \pi^{1 / 4} \frac{3}{8} \frac{a}{c_{0}} \sqrt{\frac{\delta}{D_{g b}}}(4 D)^{-1 / 4}\right)^{4 / 3}
$$

and

$$
\frac{\Delta L}{\Delta t}=\frac{d L}{d t}=\frac{a}{\left(r_{0} \pi^{1 / 4} \frac{3}{8} \frac{a}{c_{0}} \sqrt{\frac{\delta}{D_{g b}}}(4 D)^{-1 / 4}\right)^{4 / 3}}=A \frac{1}{r_{0}^{4 / 3}}
$$

To estimate the growth rate of ZnSe nanowires versus diameters, we use the following parameters: $D_{g b}=10^{-13}$ $\mathrm{cm}^{2} / \mathrm{s},{ }^{16,18} D=10^{-17} \mathrm{~cm}^{2} / \mathrm{s},{ }^{16} c_{0}=2.7$ atom $/ \mathrm{nm}^{2} ;{ }^{19}$ the lattice parameter for ZnSe is $a=0.567 \mathrm{~nm} ; \rho=21.7$ atom $/ \mathrm{nm}^{3}$. Then, $A$ is determined to be $0.5 \mathrm{~nm}^{7 / 3} / \mathrm{s}$. Comparing to the experimental data, we obtained:

$$
v=A \frac{1}{r_{0}^{4 / 3}}+C
$$

where, $C$ is about $0.012 \mathrm{~nm} / \mathrm{s}$ which means the constant deposition of the source atoms. This value is consistent with the deposition rate of $\mathrm{ZnSe}$ on a flat substrate at about $390{ }^{\circ} \mathrm{C}$ in MBE. The diameter-dependence of the growth rate estimated by equation (8) matches the experimental data fairly well (see Fig. 2 (a)). We noticed that this interface diffusion mechanism is different from that of the surface diffusion controlled mechanism. ${ }^{8}$ This is because the surface diffusivity is generally one order higher than that of

\section{References}

[1] R. S. Wagner and W. C. Ellis, Appl. Phys. Lett. 4, 89 (1964).

[2] E. I. Givargizov, J. Cryst. Growth 31, 20 (1975).

[3] E. I. Givargizov, Highly Anisotropic Crystal, D. Reidel Pub. Co., 1987.

[4] T. Y. Tan, N. Li, and U. Gosele, Applied Physics AMaterials Science \& Processing 78, 519 (2004).

[5] S. Kodambaka, J. Tersoff, M. C. Reuter, and F. M. Ross, Phys. Rev. Lett. 96, 096105 (2006).

[6] V. Schmidt, S. Senz, and U. Gosele, Phys. Rev. B 75, 045335 (2007).

[7] Y. Cai, S. K. Chan, I. K. Soar, Y. T. Chan, D. S. Su, and N. Wang, Adv. Mater. 18, 109 (2006).

[8] S. Kodambaka, J. Tersoff, M. C. Reuter, and F. M. Ross, Science 316, 729 (2007).

[9] G. Neumann and G. M. Neumann, Surface Self-Diffusion of Metals, Diffusion Monograph Series, Diffusion Information Center, 1972.

[10] Y. F. Chan, X. F. Duan, S. K. Chan, I. K. Sou, X. X. Zhang, and N. Wang, Appl. Phys. Lett. 83, 2665 (2003).

[11] Y. Cai, S. K. Chan, I. K. Sou, Y. T. Chan, D. S. Su, and N. Wang, Small 3, 111 (2007) the grain boundary diffusivity ${ }^{16}$, and for the present model, the deposition of the source atoms is mainly controlled by boundary diffusion. For the nanowires with a diameter $d>$ $60 \mathrm{~nm}$, the growth rate is very low because of the increase of the diffusion length at the catalyst interface. This dramatically lowers the deposition rate of atoms at the central area of the nanowire-catalyst interface. In real experimental condition, however, the atoms on the catalyst surface should have a high mobility and are in semi-melting state. The surface can always capture the source atoms to cause a constant growth of the nanowire. For ultrathin nanowires, the melting points of the catalysts will decrease by increasing their diameters. For the nanowires with diameters larger than $60 \mathrm{~nm}$, the directly impinging atoms on the liquid catalysts will play a dominant role during the growth, and the nanowire growth will follow the classical VLS scheme.

This work was financially supported by the Research Grants Council of Hong Kong (Project Nos. N_HKUST615/06, G_HK021/07) and partially supported by the Nanoscience and Nanotechnology Program at HKUST.

[12] Z. H. Zhang, F. F. Wang, and X. F. Duan, J. Cryst. Growth 303, 612 (2007).

[13] U. Philipose, A. Saxena, H. E. Ruda, P. J. Simpson, Y. Q. Wang, and K. L. Kavanagh, Nanotechnology 19, 215715 (2008).

[14] L. E. Froberg, W. Seifert, and J. Johansson, Phy. Rev. B 76, 15340 (2007).

[15] W. Seifert, M. Borgstrom, K. Deppert, K. A. Dick, J. Johansson, M. W. Larsson, T. Martensson, N. Skold, C. P. T. Svensson, B. A. Wacaser, L. R. Wallenberg, and L. Samuelson, J. Cryst. Growth 272, 211 (2004).

[16] N. A. Gjostein, Diffusion, edited by Aaronson, H. I. American Society for Metals, Metals Park, OH, 1973.

[17] I. Kaur, Fundamentals of grain and interphase boundary diffusion, John Wiley, 1995.

[18] I. Kaur and W. Gust, Handbook of grain and interphase boundary diffusion data, Ziegler Press, 1989.

[19] L. L. Chang and K. P. Ploog, Molecular beam epitaxy and heterostructures, Dordrecht ; Boston : M. Nijhoff, 1985. 